\title{
VALORACIÓN DEL AMBIENTE VIRTUAL DE APRENDIZAJE DE LA UNIVERSIDAD DE NARIÑO VIRTUAL
}

\author{
Por: Mónica Patricia Cerón Benavides ${ }^{1}$ \\ Coautores: Jordi L. Coiduras R. ${ }^{2}$ - Carlos A. Guazmayan Ruiz ${ }^{3}$
}

\section{RESUMEN}

La propuesta de una metodología que permita la valoración del ambiente virtual de aprendizaje desde la perspectiva del estudiante, subyacente a la propuesta de educación virtual de la Universidad de Nariño Virtual, es necesaria e imprescindible, en el sentido de lograr una visibilidad general según la experiencia merecida por el educando en estos ambientes.

No debe ser vista desde lo absoluto, sino por el contrario, como una propuesta que con el transcurso del tiempo se modifica, presta a los avances que emergen, los cambios, mestizajes de saberes, interculturalidades y diversos lenguajes que caracterizan al ser humano.

La información que se logra obtener al aplicar una estrategia valorativa del AVA a los estudiantes, permite desde una postura crítica: revisar la adecuación y pertinencia de los procesos de seguimiento y evaluación institucional, favorecer la aproximación a la visión y experiencia de los destinatarios, la identificación de las debilidades para convertirlas en oportunidades de mejora. Todo ello es coherente y responde a la misión de la Universidad de Nariño, comprometida con una educación superior de calidad.

Palabras clave: Ambiente Virtual de Aprendizaje, AVA, Educación Virtual, Valoración.

\section{Clasificación JEL: 121}

1. Licenciada en Informática (U. de Nariño). Investigadora grupo e-TIC. Máster en Tecnología Educativa y Gestión del Conocimiento (U. de Lleida). Colombiana. Email: monpatik@hotmail.com

2. Doctor en Psicopedagogía. Docente de la Universidad de Lleida-España. Email: oiduras@pip.udl. cat

3. Mg. en Pedagogía de l' informátic (U. de Lovaina), Doctor en Educación (UB), Director Universidad de Nariño Virtual. Email: cgubes@yahoo.es

Artículo recibido: 19 de julio 2014.

Aprobación definitiva: 30 de septiembre de 2014. 


\title{
VIRTUAL LEARNING ASSESSMENT AT THE VIRTUAL UNIVERSITY OF NARIÑO
}

\author{
By: Mónica Patricia Cerón Benavides \\ Coautores: Jordi L. Coiduras R. - Carlos A. Guazmayan Ruiz
}

\begin{abstract}
The purpose of this paper is to propose a methodology for the evaluation of a virtual learning environment from the perspective of the student. The previous statement underlines the proposal of virtual education from the Virtual University of Nariño as it is necessary and essential, in the sense of achieving overall visibility in the experience earned by learners in these environments.

This methodology should not be seen as a whole, but on the contrary, as a proposal that can be modified over time. It is prepared to face the emerging developments, changes, crossbreeding of knowledge and different languages and cultures that characterize every being.

The information obtained from applying an evaluative strategy to students allows from a critical stance: review the adequacy and relevance of the monitoring processes and institutional assessment to promote the vision and experience of the users to identify weaknesses and to turn them into opportunities for improvement. All this is consistent and meets the mission of the University of Nariño, which is committed to a quality higher education.
\end{abstract}

Key Words: Virtual Learning Environment, AVA, Virtual Education, Assessment. JEL Classification: 121 


\section{Introducción}

Actualmente, en el marco del desarrollo de políticas educativas tendientes a gestionar una educación inclusiva que vaya mucho más allá de ampliar la cobertura de la educación superior, en Colombia se ha dado un especial impulso a los programas diseñados en el paradigma de la educación virtual. La Universidad de Nariño Virtual (UdenarVirtual), ha generado una oportunidad educativa especialmente para jóvenes y adultos tradicionalmente aislados geográficamente y en condiciones de vulnerabilidad social, garantizándoles el acceso a la educación superior que mejore su calidad de vida en todos los órdenes, contextualizada desde lo regional, toda vez que el paradigma de la educación virtual facilita la gestión financiera y logística para su desarrollo.

En el convenio suscrito entre la Universidad de Nariño y el Ministerio de Educación Nacional de Colombia MEN, en el 2011, para la creación de programas de pregrado virtuales, se diseñó una metodología con sus respectivos instrumentos, para validar, fundamentalmente los módulos: Cultivo de cacao, Técnicas para la siembra, Renovación y propagación de cacao, y Viveros de cacao. El proceso concluye con resultados finales aceptables y por ende módulos validados.

El objetivo es, por lo tanto, la consolidación de una metodología que reconozca la valoración, no de módulos, sino de todo el Ambiente Virtual de Aprendizaje presente en el Campus Virtual de la Universidad de Nariño. Se debe entender la valoración como un proceso de retroalimentación efectiva que permite obtener información cualificada a partir de los principales protagonistas: los estudiantes, en relación a las características de los procesos desarrollados en las diversas situaciones de aprendizaje, su validez, pertinencia y calidad, que se evidencia y analiza holísticamente en el semestre académico vigente de un programa de formación virtual.

De tal manera, que la Propuesta para la Valoración del Ambiente Virtual de Aprendizaje de la UdenarVirtual, pretende gestionar, por un lado la búsqueda de altos parámetros de calidad sobre los elementos virtuales construidos y facilitados en el programa ejecutando un proceso de información, comunicación y retroalimentación complejo, requerido por el equipo transdisciplinar y las distintas instancias académico/administrativas para fundamentar decisiones en relación a políticas educativas y criterios de calidad en educación virtual; y por otra parte, asentar las bases conceptuales e instrumentales para la validación de dichos elementos y los distintos panoramas virtuales que se suscitan en el proceso formativo.

\section{Fundamentación conceptual}

La nueva relación del lazo social potencializada por las TIC, permite entre otras cosas, la democratización del saber, la versatilidad de la inteligencia nómada y la producción de saberes significativos con sentido social, por ende, el desarrollo integral del ser humano a partir de los cuales la educación virtual se puede sustentar con mayor relevancia. La pedagogía y la didáctica que emergen de este paradigma educativo pueden labrar uno de tantos caminos para lograr calidad y pertinencia de la educación superior, contextualizadas con las necesidades, características y ritmos de los estudiantes. 
Se considera que la valoración del ambiente virtual de aprendizaje, se constituye más que en un dato, en un proceso de calidad de gran valor, ya que, la UdenarVirtual elabora la planeación del currículo por ciclos propedéuticos, que consiste en ciclos secuenciales y complementarios divididos por semestres, cada uno de los cuales tiene titulación, hasta llegar al título profesional. Se desarrolla la malla curricular en base a dos elementos: el primero la organización de los cursos alrededor de un concepto articulador para cada semestre, y segundo, la organización por componentes de formación según el tipo de competencias de los cursos que hacen parte del semestre académico. De tal manera, que los cursos se organizan para otorgarle integración al currículo, sobre un "concepto articulador" como eje integrador de todos los contenidos que se van a desarrollar en cada semestre. Vislumbrando así, la interdisciplinariedad que va aún, más allá, pues genera un acercamiento multidisciplinario, en donde los diversos contenidos son nucleados en torno a ejes conceptuales y prácticos.

Para llevar a buen término, en primer lugar, el propósito de la valoración de las categorías subjetivas, estas son las siguientes:

Visión Ética. El desarrollo de las competencias relacionadas con el ser humano, se pretende describir cómo los estudiantes aplican en condiciones de contexto y de su vida cotidiana, los conocimientos gestionados, y si la estrategia avanza hacia el aprendizaje autónomo, cooperativo y colaborativo en los AVA y en la Red (la web). Como dice Freire (2001) la educación es política, y por tanto tiene una intencionalidad individual y colectiva, determinada por valores éticos que se encuentran en el objetivo de la acción educativa.

La virtualidad implica una nueva forma de alteridad y necesita por tanto una recontextualización de viejos valores y políticas. "Existe una presencia ética en la virtualidad. La virtualidad, concretada en espacios de interacción, la formamos personas, seres humanos capaces de sentir y de manifestarnos valorativamente, en otras palabras, sujetos éticos" (Duart, 2003, p. 3).

Según lo reformula Morín (1990), se requiere de nuevos pensamientos y conocimientos para interpretar, comprender lo que de porvenir tiene el mundo globallocal. Las comunidades virtuales se presentan como un no lugar, donde navegar por Internet, re-crea el comportamiento del nuevo ciberciudadano interconectado. Las nuevas formas de relacionarse y de saber, generan un choque entre paradigmas y nos retrotrae al imaginario del malestar, huimos, esa crisis de valores, invita a que es tiempo de repensar el Mundo de la Vida, bajo narraciones distintas de bienestar.

Visión Estética. Relacionando lo que menciona Barbero (1996), se concibe la estética como el espacio donde se construye un nuevo paradigma de la relación entre la lógica del discurso y lo visible (la forma), de la inteligibilidad y la sensibilidad. La estética de la educación virtual es así, la representación abstracta por medio de la relación persona-ordenador-sociedad, de una realidad construida desde la imagen y para la imagen, lenguajes y escrituras distintas de pensamiento, aprendizaje y enseñanza.

Un paradigma que cuestiona radicalmente lo lineal y transmisible, que enaltece las prácticas e intertextualidades, un saber mosaico, móvil y con fronteras divi- 
sibles, reestructura el sistema comunicativo, donde se necesita el rigor de pensar la Ciencia y Tecnología pero teniendo en cuenta, articulando y enriqueciendo la experiencia cultural, la reflexividad, la intuición, nuevas sensibilidades, otros saberes, otras formas de sentir, de relacionarse y reconocerse en la relatividad del tiempo y el espacio.

Visión de Visibilidad. Con ella se busca entender y describir cuál es la imagen que el estudiante construye de sí mismo, en la práctica y gestión profesional de la educación virtual. La forma como los estudiantes se aceptan y entienden en dicho contexto, es uno de los cambios más estructurales en el nuevo paradigma educativo virtual.

Las relaciones visibles e invisibles de la multiplicidad de seres entre la vieja denominación, naturaleza-cultura, mundo-hombre, o estructura física-bióticaantrópica, como lo identifica Guazmayan, (2003), traduce la captación de elementos heterogéneos articulados a un sistema de interdependencia. La innovación busca aliados, portavoces, para multiplicar los interlocutores de las redes.

El entrecruce de mestizajes (saberes e identidades) provocan un nuevo orden caótico (oralidad, literalidad, sensibilidad, simbolismo) que emergen en la cibersociedad, lo cual puede facilitar o entorpecer el proceso de enseñanza aprendizaje, sino se mira desde una forma distinta al instruccionismo; cautivando el goce de aprender y la idea de aprender porque se desea, y no porque un sistema de grados y requisitos institucionales lo obligan.

Visión de Sensibilidad. Con ella se pretende describir la motivación intrínseca del estudiante al formar parte de una propuesta formativa de educación virtual superior.

La neutralidad de los tecnócratas que reducen la ciencia y la tecnología a un único campo neutral de tecno-ciencia para agenciar un estatus jerárquico burocrático emulando la lógica de la economía mercantilista, tiene que ser desmontada por una nueva democracia en tiempo real, valorizando la vida real de los individuos, los grupos y los colectivos.

La actual escuela representa el secuestro de la imaginación y la consagración de la banalidad, pero mediante los mass media, el internet, la hipermedia, la transmedia, puede convertirse, refiriendo a Barbero (2002), en un espacio no solo de apropiación sino también de descubrimiento crítico de los contenidos que se transmiten, de los cambios en la experiencia social y de la narrativa cultural que esos medios catalizan. Nuevas y distintas formas de percibir, sentir, relacionarse, construir la imagen personal y social interactuando tanto en el espacio geográfico y virtual a través de las TIC.

La educación debe propender y el estudiante percibir que la motivación además de ser la energía intrínseca en sí mismo, también confluye en la multiplicación de los encuentros, la red social, en el juego del hipertexto, la multimedia y el contexto en los procesos de aprendizaje en el ciberespacio.

Lo anterior, fundamenta la validación del ambiente virtual de aprendizaje sobre expresiones cualitativas, en cohesión con ellas, se presenta en segundo lugar, pero no por eso menos importante, los preceptos que sustentan la valoración de las 
categorías físicas-tecnológicas, aspectos que se caracterizan por ser más fáciles de percibir físicamente, por cuanto son tangibles y/o cuantificables. Se estudia y analiza la capacidad instalada y el talento humano que posee el ambiente de aprendizaje en un semestre del programa virtual. Por lo tanto, la segunda sección de la encuesta se enfoca a identificar los componentes según Silvio (2000) de: infraestructura física, infraestructura lógica, actores, recursos, servicios telemáticos y el software de aplicaciones individuales.

El computador, no es un instrumento con el que se producen objetos (a pesar que las impresoras de más reciente generación ya "imprimen” objetos), ni una deidad que por sí sola inventa y concibe aprendizaje, sino un recurso tecnológico de gran importancia para el procesamiento de la información y el desarrollo de las TIC, pero desde la fundamentación pedagógica y el paradigma que cada persona o institución argumenta. Desde la virtualidad, este es un artefacto que facilita la descentralización de las formas de transmisión y transformación mediática, la comunicación y la gestión del conocimiento social, reconfigura o potencializa distintas formas de manifestar sentimientos y reflexión, da pie a una mente distinta que intenta descifrar e innovar estrategias de enseñanza y el aprender, reconstituyen un ámbito de socialización, de proyección, de competencias y estilos de vida.

Las visiones cualitativas funcionan sinérgica y holísticamente con los componentes tecnológicos y humanos, estos son:

Infraestructura física: pretende obtener información sobre los equipos disponibles que el estudiante puede tener y acceder, ya sean personales, institucionales o comerciales, para el procesamiento de la información y gestión del conocimiento y la conectividad.

Infraestructura lógica: abarca componentes lógicos básicos: software imprescindible para el funcionamiento de los dispositivos físicos como servidores y la red física para la conectividad a internet que la universidad posee, posibilitando el tratamiento/generación/publicación de información y comunicación a través de la plataforma de aprendizaje virtual.

Actores: se refiere al personal humano que utiliza y aprovecha el hardware y software para lograr la comunicación e interacción entre los mismos, el tutor y el estudiante, el estudiante con sus compañeros, con expertos externos, con el personal administrativo, directivo y de soporte web de la UdenarVirtual.

Recursos: hacen referencia a aquellos elementos didácticos con los que puede contar el estudiante para el apropiado desarrollo de las competencias, temáticas y contenidos.

Servicios Telemáticos: son parte de la infraestructura lógica de las redes, pero que cumplen en ella una función especial, facilita la interfaz directa de comunicación entre los usuarios o entre usuario y la red-internet.

Software de aplicaciones individuales: hace referencia a programas básicos y programas especializados que el usuario de manera local, privada o personal, utiliza para producir sus propios contenidos, aquellos que posteriormente comparte en la red. 


\section{Metodología}

Se trabajó con una metodología mixta, entendida como hibridación de técnicas tomadas de la metodología cuantitativa y de la cualitativa. En la metodología cuantitativa, se da mayor relevancia a lo medible, cuantificable, busca los hechos o causas de los fenómenos sociales, es particularista: las partes representan al todo (deductiva); sin prestar atención a lo subjetivo de cada individuo. Según Hueso y Cascant (2012), Se basa en el uso de técnicas estadísticas para conocer ciertos aspectos de interés sobre la población que se está estudiando (p. 7).

La metodología cualitativa, basa su análisis en la reflexión de la intersubjetividad de los significados, símbolos e interpretaciones que manifiesta el sujeto en contexto, pues estas desde la praxis e interacción con los demás constituyen y crean la realidad estudiada. Como señala Pérez Serrano (1990), "No existe una única realidad, sino múltiples realidades interrelacionadas”.

Se diseña, valida y aplica la encuesta a todos los estudiantes de tercer semestre del programa Técnico Profesional en Agroindustria Alimentaria; para luego organizar y analizar los datos mediante escala de actitudes Likert.

\section{Resultados}

De manera muy breve se presentan los resultados obtenidos en la investigación:

Gráfica 1

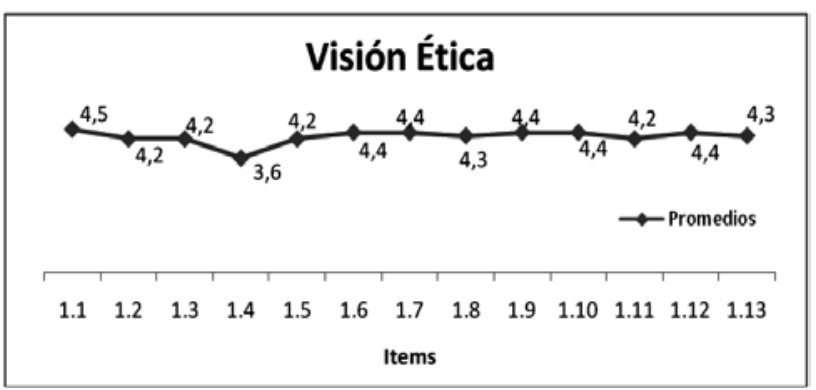

Fuente: esta investigación

La mayoría de los estudiantes asume una actitud muy favorable frente al trabajo individual, y al fomento de este en su entorno de aprendizaje, no solo, se desarrolla actitudes de responsabilidad individual, sino que se trabaja de manera articulada con la metodología colaborativa, el trabajo en equipo, dejando ver con esto la importancia que presta el AVA a través de actividades específicas en un modulo virtual, para el desarrollo de estas habilidades y competencias en el estudiante. 


\section{Gráfica 2}

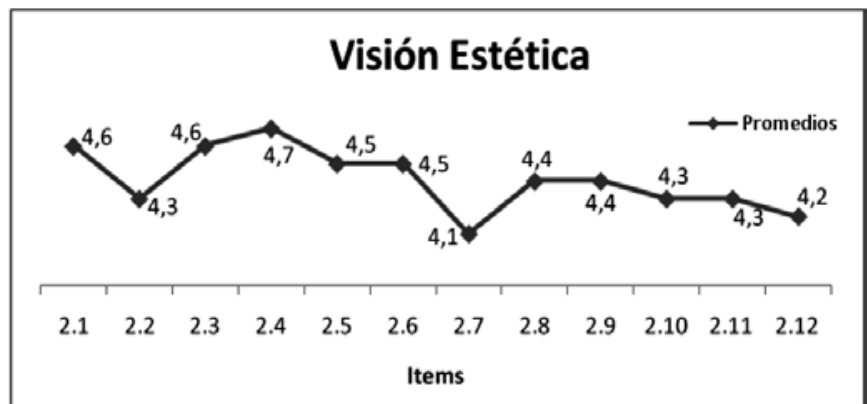

Fuente: esta investigación

Se evidencia una buena articulación entre los distintos saberes, no se incluye solo el conocimiento científico, o todo lo contrario, sino que se trabaja tomando lo mejor de cada uno de ellos y respetando los mismos para construir realmente aprendizaje significativo. El AVA le permite construir juicios propios útiles no solo para sí mismo sino también para la sociedad.

\section{Gráfica 3}

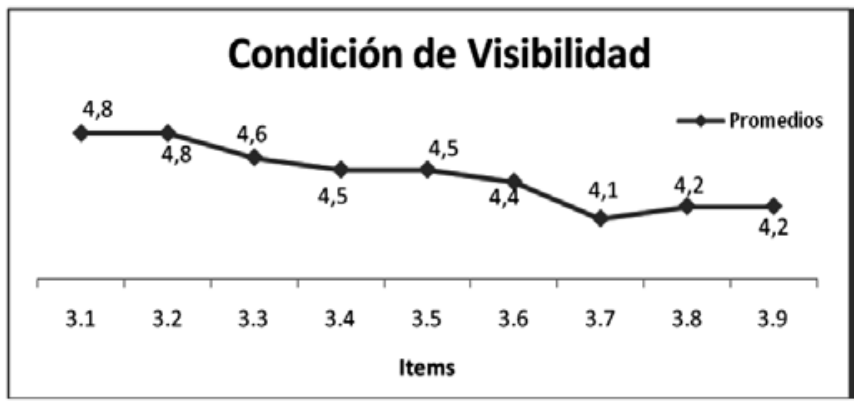

Fuente: esta investigación

Los estudiantes valoran este aspecto de manera adecuada, lo que permite inferir que los estudiantes trabajan y van por el buen camino, lo cual se traduce en la captación de elementos heterogéneos articulados a un sistema de interdependencia que hacen de ellos seres visibles en la red. En la medida en que ellos, de manera autónoma, hacen uso de esta innovación, ellos buscan aliados, se convierten en portavoces, para multiplicar los interlocutores de las redes, generando un nuevo tejido social en el ciberespacio. Se asume una actitud favorable frente al AVA. Se ven más que un profesional, se visualizan como seres humanos que concibe la importancia de actuar sobre valores y ética apropiada para bien de la sociedad. 


\section{Gráfica 4}

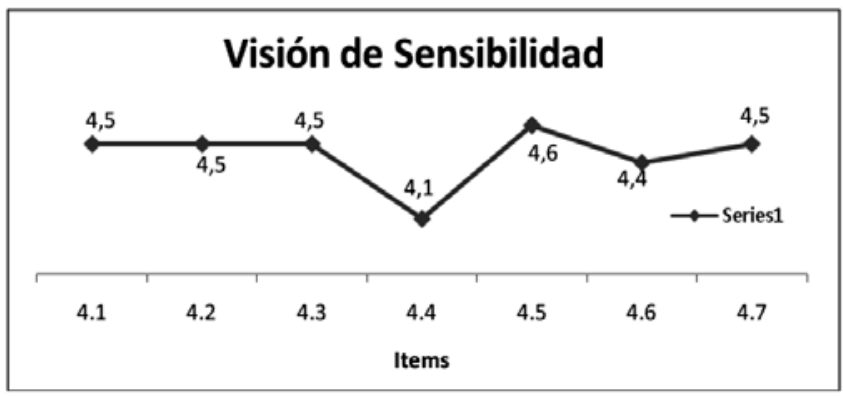

Fuente: esta investigación

Entre las opiniones de los estudiantes la UdenarVirtual, ha sido una gran oportunidad para cumplir sus metas de reconocimiento como personas valiosas dentro de sus territorios y contextos que les permite diseñar y desarrollar sus proyectos de vida. De tal manera que se aprecia relación coherente entre las observaciones descritas y las valoraciones de los diversos ítems registrados en esta primera sección de la encuesta.

En relación a la valoración de las categorías de infraestructura tecnológica y humana del ambiente virtual de aprendizaje, se obtuvo:

\section{Gráfica 5}

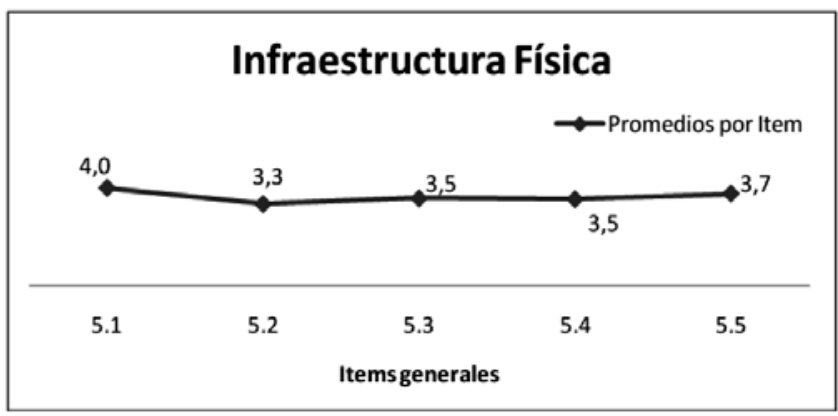

Fuente: esta investigación

La disponibilidad de equipos (portátiles y de interacción) y acceso a internet, son adecuados a pesar de los inconvenientes geoespaciales que los caracteriza. Se logran realizar las prácticas pero se requiere una mayor inversión o disponibilidad de esta infraestructura para mejorar el ambiente práctico. Las zonas rurales carecen de las grandes ventajas de inversión que si poseen las zonas urbanas. 


\section{Gráfica 6}

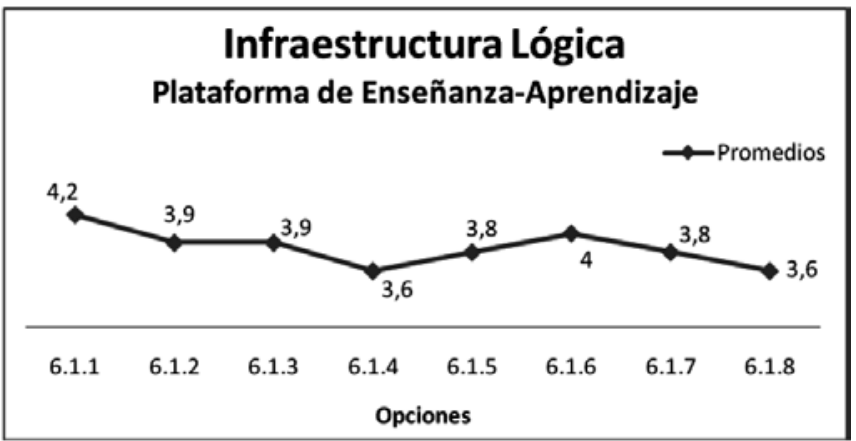

Fuente: esta investigación

La plataforma Campus Virtual UDENAR, como espacio que facilita encuentro formal de todos a todos en el ciberespacio, se caracteriza por lograrlo de manera adecuada y agradable; ha facilitado la realización de actividades, en distintos formatos en tiempo real y diferido, entre otros aspectos. A pesar de encontrar algunas manifestaciones de pequeño inconformismo de los estudiantes, respecto al límite de espacio para envío de archivos.

\section{Gráfica 7}

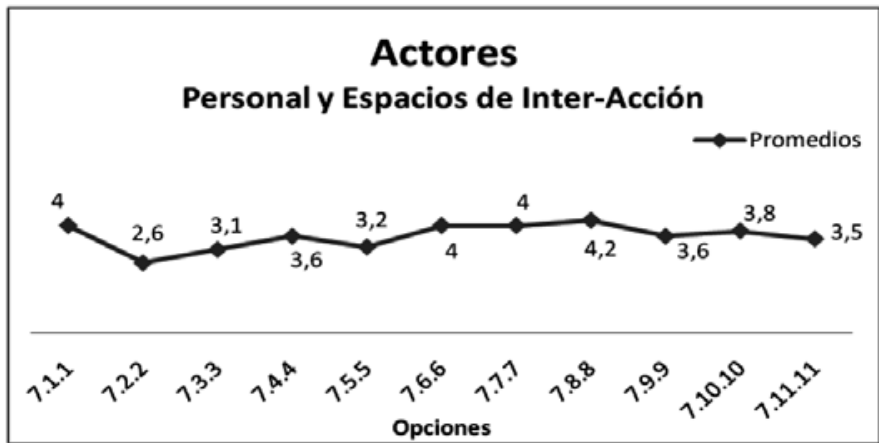

Fuente: esta investigación

Los tutores, estudiantes, expertos, administrativos, organizaciones e instituciones que logran comunicar, aplicar, orientar y atender de manera articulada a nivel académico, pedagógico, administrativo y tecnológico. Entre los ítems evaluados solo dos presentan alguna falla o cierto nivel de inconformismo: atención psicosocial, y la comunicación entre administrativos y estudiantes, de tal manera que se sugiere se reestructure o se revalué según las estrategias implementadas en la unidad, ya que no son lo suficientemente adecuadas para los estudiantes. 


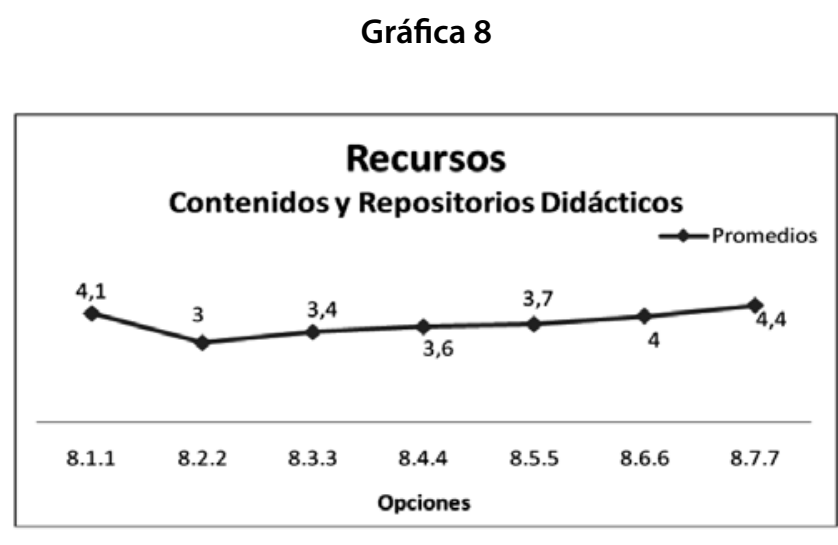

Fuente: esta investigación

En cuanto a los elementos didácticos no solo internos, sino también externos, para el apropiado desarrollo del AVA, se obtuvo que, estos se valoran de manera adecuada, aun cuando deberían facilitarse más recursos de tipo externos u orientaciones para su uso, de tal modo que pueda lograrse una mejor administración de la información.

\section{Gráfica 9}

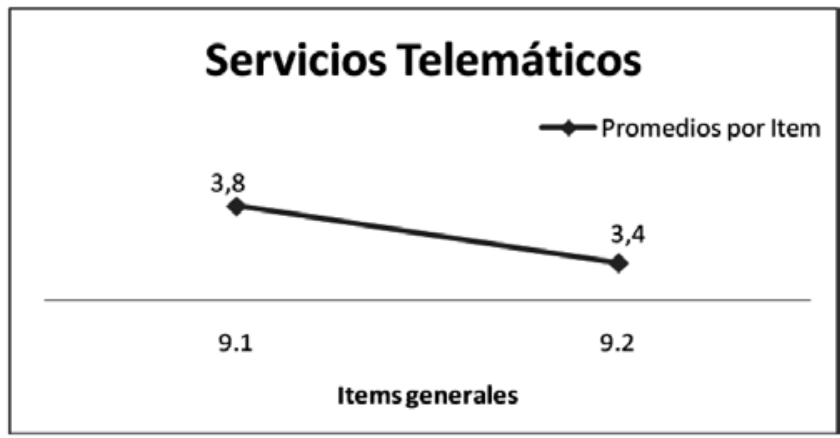

Fuente: esta investigación

Entre estos se encuentran los Servicios de comunicación, los cuales se consideran de manera adecuada, pero se evidencia una valoración baja respecto a espacios para la comunicación y respuesta oportuna a quejas/reclamos/dudas, por cuanto no existe una adecuada administración de los mismos que presten espacios de atención al usuario, siendo estos fundamentales. Búsqueda de Información y Navegación: como recursos de apoyo extras, y en complemento con anteriores valoraciones, se muestra de igual manera un promedio bajo con el ítem: Motores de búsqueda especializados, Bibliotecas y bibliografías virtuales, los estudiantes corroboran que este tipo de recursos, materiales ya sean propios o externos, uti- 
lizados en forma de consulta y aprovechados para búsquedas específicas, no son tenidos en cuenta en mayor nivel.

\section{Gráfica 10}

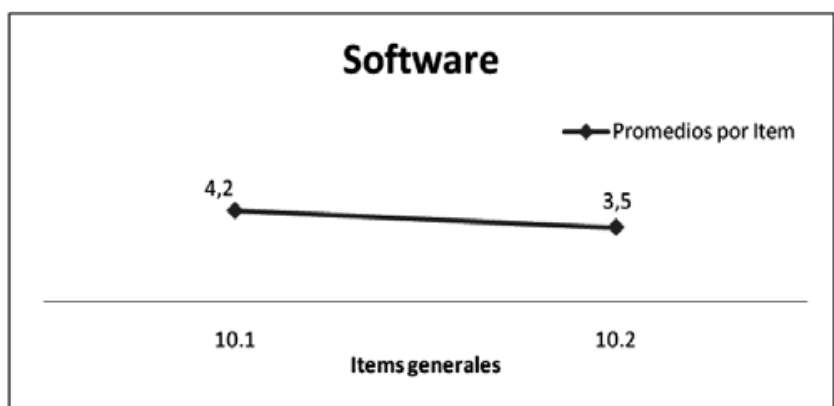

Fuente: esta investigación

Se vislumbra un buen nivel de utilidad y aplicación de los distintos programas de ofimática básicos para la realización de trabajos. Lo cual significa que los conocimientos que poseen los estudiantes no generan dificultades extremas para el uso del computador.

No se exige un nivel tan profundo de conocimiento en software especializado, por ende los resultados registrados por los estudiantes no afectan la calidad o las actividades plafinicadas, ya que estos se profundizan durante el proceso y adquieren sentido para los estudiantes, por ende se valora de manera adecuada.

\section{Conclusiones}

La mayoría de los resultados analizados en la primera sección de la encuesta, muestran puntuaciones sobre la escala Lickert, superiores a 4 puntos, lo que supone altos niveles de acuerdo con las afirmaciones propuestas. Los estudiantes creen, viven y se apropian de la experiencia de aprendizaje virtual, vislumbran y sienten su pertinencia en el contexto más cercano de su proyecto de vida.

Los escenarios de aprendizaje presentan las condiciones mínimas y necesarias, motivan y exigen de sí mismos ir más allá, no permitir conformarse, sino superarse.

Sigue expresándose por parte de algunos estudiantes la necesidad de algún contacto presencial para desarrollar o guiar experiencias de aprendizaje. El papel del profesor como asesor, motivador y colaborador (Álvarez, 2008) puede verse reforzado con la comunicación y el intercambio mediante la plataforma si se contempla la posibilidad de ofrecer espacios cara a cara, aprovechando las herramientas de comunicación IP -voz y videoconferencia- que podrían enriquecer el asesoramiento, como ofrecen algunas universidades virtuales en horarios limitados. Así como la tutoría entre iguales, por medio de foros y con la promoción de encuentros 
orientados a compartir interpretaciones en relación a las tareas, las posibilidades de resolución, entre otras temáticas.

Se puede observar algunos puntos aislados, estos, como experiencias puntuales, relacionan aspectos que han afectado la imagen de la unidad virtual (bajo nivel) y el proceso de aprendizaje en general. Pueden explicarse estos resultados como en otros lugares, por la escasa formación pedagógica del profesorado universitario y, por tanto, de los recursos de acompañamiento (Coiduras et al., 2008; Lobato et al., 2005).

Siempre actuar, y no limitarse a presentar y/o solicitar elaboración de textos, contemplar otros lenguajes y medios, tratar de saltar entre todas estas posibilidades y no quedarse en lo común y fácil, que ya conocemos como: documentos (resúmenes), seguir resaltando la evidencia de prácticas en su entorno, es muy significativo, además el hecho de que partan de conocimiento problematizado hace la diferencia pedagógica independientemente del recurso a utilizar.

En formación superior y continua se cree necesario impulsar un pensamiento de orden superior como propone Barnett (1992); en Brockbank \& McGill (2002), considerando a los estudiantes como investigadores, con tareas de análisis, síntesis, de evaluación, creación y utilizando diversas formas de representación - multilenguajes en sus producciones.

El reto más importante, innovar. Entre los resultados tabulados, aunque se evidencia el esfuerzo, se observa algunas inconformidades en relación a ello. Se incentiva al tutor a pensar las actividades, siempre con el fin de facilitar al estudiante la posibilidad de reconstruir y retroalimentar su producción.

En la segunda sección de la encuesta, en la cual se valoran condiciones tangibles, físicas, tecnológicas y recursos humanos, se determina un nivel menor en el cálculo de los promedios, en comparación con la sección primera, aquí, no difieren en gran medida pero se aprecia resultados menores a 4.0, que podemos considerar altos.

Teniendo en cuenta que la gran mayoría de los estudiantes de la UdenarVirtual pertenecen a zonas rurales del Departamento de Nariño, no es de mayor sorpresa encontrar falencias a nivel tecnológico y por ende de accesibilidad o conectividad a la plataforma para el envío y recepción de materiales, actividades, asesorías. Pero esto, no es un problema que se convierte en excusa para desmejorar la calidad educativa, sino lo contrario. Se debe tener en cuenta tales características para facilitar el proceso de producción de elementos, de tal manera que sean de mayor simplicidad para el estudiante, por ejemplo: archivos bien elaborados pero de menor tamaño (depende situación tecnológica y geográfica), material multimedia de corta duración, búsqueda especializada para agilizar y obtener resultados certeros, disponer de distintos medios de comunicación, no solo internet: tv, radio, llamadas.

Sobre el análisis general de los resultados, se aprecia que existe y se identifica la relación entre los diversos módulos que componen un semestre académico, la organización de estos sobre la base de un concepto articulador como eje integra- 
dor de todos los contenidos, deja ver que es una estrategia curricular que genera agradables experiencias, la valoración del Ambiente Virtual de Aprendizaje, es un todo, y por ello la interdisciplinariedad y la filosofía de educación virtual se concibe por buen camino, según la perspectiva del estudiantado.

$\mathrm{Al}$ ser un ambiente virtual de aprendizaje en donde todos los elementos conforman un todo, significa, que si algo falla, los demás deben saber actuar de manera acorde a las necesidades para solventarlas, y no solo en la dimensión pedagógica, sino en cada una de las existentes: administrativa, comunicativa, tecnológica. Estas dimensiones en armonía se interrelacionan para trabajar sobre un propósito en común, armar un ambiente de aprendizaje en donde el principal protagonista de este, sea el estudiante. Por ello, es indispensable continuar evaluando y autoevaluando cada dimensión que conforma la unidad virtual, y ante el hecho de no registrar datos totalmente al $100 \%$ de la valoración, indica que existen falencias, así sean mínimas deberán ser evaluadas; sobre todo considerando que la educación es un proceso que exige la revisión cíclica, continua, para lograr una mayor eficacia y calidad, por cuanto los actores manifiestan cambios en el tiempo. 


\section{Referencias}

1. ÁLVAREZ, M. (2008). La tutoría académica en el espacio europeo de la educación superior.

2. BARBERO, J. (1996). Heredando el futuro. Pensar la educación desde la comunicación. Recuperado el 20 de Julio de 2013, en: http://www.redalyc.org/pdf/1051/105118998002. pdf

3. BARBERO, J (2002). La educacion desde la Comunicación. Recuperado el 20 de julio de 2013, en: http:// www.eduteka.org/pdfdir/SaberNarrar.pdf

4. BARNETT, R. (1992); en BROCKBANK, A. \& MCGILL, I. (2002).

5. COIDURAS, J.; CARRERA, X. ET JOVÉ, C. (2012). Le portfolio électronique comme espace d'ccompagnement et de confrontation avec l'expérience dans des processus de formation en alternance. Revue TransFormations, 7; pp. 79-92. Lille: Université de Lille.

6. DUART, J. (2003). Educar en valores en entornos virtuales de aprendizaje: realidades y mitos. Recuperado el 20 de julio de 2013, en línea: http://www.uoc.edu/dt/20173/20173.pdf

7. FREIRE, P. (2001). Política y educación. Quinta edición. Buenos Aires. Siglo XXI.

8. GUAZMAYAN, C. (2003). Documento principal Universidad de Nariño Virtual, Modelo de equilibrio. Recuperado el 1 de septiembre de 2013, en línea: http://www.google.com.co/url?sa=t\&rct=j\&q=

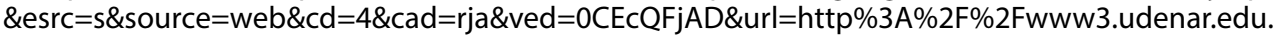
co\%2Fviceacademica\%2Fproy_men\%2FABRIL\%2FUVIRTU.doc\&ei=sHRHUo3pL4T- 9QSynoGoDA\& usg=AFQjCNGWQ3TwvaDxRrp51KJuEPB5h_YfuA\&sig2=XqN9Qimj4eAuQVeRwyVGlg\&bvm=bv.53 217764,d.eWU.

9. GUAZMAYAN, C. (2004). Internet y la Investigación Científica. El uso de los medios y las nuevas tecnologías en la educación. Recuperado el 13 de septiembre de 2013, en: http://books.google.com.co/books?id =eqFBTolXw9MC\&pg=PA75\&lpg=PA75\&dq=regeneran+continuamente+la+red+que+los+ha+prod ucido\&source=bl\&ots=zSTDRvhdVs\&sig=mX3Fm5TBC4uXOW1cDRHHypIDNo\&hl=es\&sa=X\&ei=DxyUsnbNM6jkQep3YDAAw\&ved=0CCwQ6AEwAA\#v=onepage\&q=regeneran $\% 20$ continuamente $\% 20$ la\%20red\%20que\%20los\%20ha\%20producido\&f=false

10. HUESO, A.Y CASCANT, J. (2012). Metodología y técnicas cualitativas de investigación. Recuperado el 30 de noviembre de 2013,en:http://riunet.upv.es/bitstream/handle/10251/17004/Metodolog\%C3\%ADa\%20 y\%20t \%C3\%A9cnicas\%20cuantitativas\%20de\%20investigaci\%C3\%B3n_6060.pdf?sequence=3

11. SILVIO, Jose. (2000). La Virtualización de la Universidad. ¿Cómo transformar la educación superior con la tecnología? Caracas. Recuperado el 22 de julio de 2013, en: http://www.schoolofed.nova.edu/dll/ spanish/modulos/conocimiento/Silvio_La_virtualizacionuniv.pdf

12. MORIN, E. (1990). Introducción al pensamiento complejo. Recuperado el 04 de agosto de 2013, en: http://www.edgarmorin.org/images/publicaciones/edgar-morin-introduccion-al-pensamientocomplejo.pdf

13. PÉREZ SERRANO, Gloria (1990). Investigación cualitativa. Retos e interrogantes (pdf). Recuperado el 3 de julio de 2013, en: https://psicologiaysociologia.files.wordpress.com/ 